\title{
Evaluation of Tube Wall Thickness of Feed Water Heater*
}

\author{
Takahisa UCHIKURA**, Koichi MORISAKI** and Seiichi HAMADA** \\ ** Thermal Power Plant Engineering Center Thermal Power Department \\ TOKYO ELECTRIC POWER COMPANY, \\ 1-3-3 UCHISAIWAI-CHO, CHIYODA-KU, TOKYO,JAPAN \\ E-mail:uchikura.talahisa@tepco.co.jp
}

\begin{abstract}
With regard to the high pressure(HP) feed water heater of thermal power plant at Tokyo Electric Power Company(TEPCO) sites, inspection of feed water(FW) tubes wall thickness are conducted whenever required such that frequent tube leak occurs. As a standard inspection methodology, FW heater is disassembled during planned outage, tube wall thickness is measured by the ultrasonic pulse techique(UT), then plugs are installed at the both ends of FW tube if its measured wall thickness is found below calculated threshold.However, the root causes of wall thinning of FW tube are various such as erosion and corrosion, based on wall thinning condition, the above threshold is not applied but utilizing the other technically well-grounded evaluation method is sometimes more rational.Therefore, TEPCO classified wall-thinning condition based on inspection data and established technically well-grounded and rational evaluation methodologies of $\mathrm{FW}$ tube wall thickness to suite each wall thinning condition. Moreover, with recent improvement of inspection technique, technology enabled faster, larger amount, and more accurate data acquisition, TEPCO has developed the systematized evaluation methodology that can transact data acquisition and evaluation simultaneously.This article introduces the logic of evaluation methods and examined algorithms to make them systematized.
\end{abstract}

Key words: Feed Water Heater, Tube Wall Thickness, Logic of Evaluation Methods

\section{Introduction}

A feed water heater is facility which extracts steam from the middle stage of a turbine, and heats the water supply to a boiler gradually in order to raise the thermal efficiency of a thermal power plant. Although a feed water heater has a surface heating type and a mixed heating type, in TEPCO's plant, the general surface heating type is used as a feed water heater. The feed water heater is classified into the Low Pressure(LP) water heater and the HP feed water heater in the position before and behind the feed water pump of a water system. 14 feed water heaters per one unit are installed in the plant of TEPCO. For example, the HP feed water heater of 1,000 MW of super-critical pressure unit has about 2,000 carbon-steel heating tubes the length of which are $20 \mathrm{~m}$, and the outside-diameter $16 \mathrm{~mm}$. Inspection of $\mathrm{FW}$ tubes wall thickness are conducted whenever required such that frequent tube leak occurs. As a standard inspection methodology, FW heater is disassembled during planned outage, tube wall thickness is measured by UT, plugs are installed at the both ends of FW tube if its measured wall thickness is found below calculated threshold. However, if the wall-thickness data of the perimeter is taken into consideration even if the wall-thickness value of one of points is less than the predetermined threshold, in the neighborhood, the strength of the heating tube is fully maintained in many cases. When the 
above usual criteria are adopted, even the heating tube which is maintaining still sufficient strength will be judged to be "NO GOOD", and excessive repair will be carried out. The root causes of wall thinning of FW tube are various such as erosion and corrosion, based on wall thinning condition, the above threshold is not applied but utilizing the other technically well-grounded evaluation method is sometimes more rational.

Therefore, TEPCO classified wall-thinning condition based on inspection data and established technically well-grounded and rational evaluation methodologies of FW tube wall thickness to suite each wall thinning condition. Moreover, with recent improvement of inspection technique, technology enabled faster, larger amount, and more accurate data acquisition, TEPCO has developed the systematized evaluation methodology that can transact data acquisition and evaluation simultaneously.

This article introduces the logic of evaluation methods and examined algorithms to make them systematized.

\section{Evaluation Technique}

The Japanese regulatory rules require "the structure of a pressure-proof portion must be safe to the maximum stress generated at a maximum allowable working pressure or maximum allowable working temperature. In this case, the stress produced into a proof-pressure portion must not exceed the allowable stress of the material used for the portion concerned".

The fundamental view of this evaluation method argues about safety on the basis of the allowable stress which considered the suitable safety factor for strength, such as tensile strength and a yield strength. As mentioned above, the thinning factor of the heating tube of a feed water heater is various. It evaluates by classifying into general thinning which should be directly evaluated using the required minimum thickness (threshold), local thinning which can be indirectly evaluated using the required minimum thickness, and the pitting corrosion which can be evaluated regardless of the required minimum thickness.

\subsection{Characteristics to be inspected}

Characteristics to be inspected should be the wall-thickness values of heating tubes.

\subsubsection{Wall-thickness measuring methods ${ }^{[1]}$}

A wall-thickness measurement by the ultrasonic pulse techique from the inside of the tube(IUT) is performed for the carbon-steel pipe which is a ferromagnetic substance. Fig. 1 shows the schematic diagram of the IUT principle.

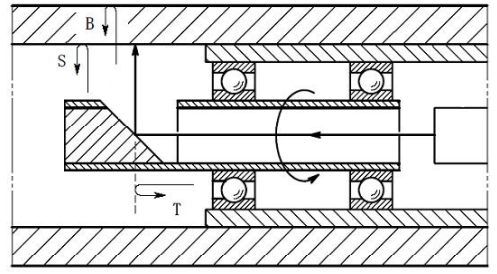

(a)

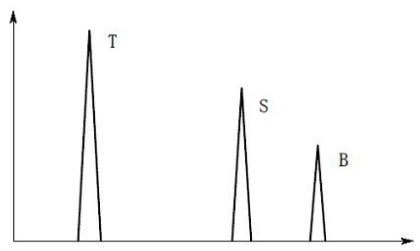

(b)

Fig. 1 Schematics of the IUT principle 
Table.1 Specifications of Tubes Measurement pitch

\begin{tabular}{|l|l|c|}
\hline \multirow{3}{*}{ Feed water heater tube } & material & JIS G3456 STPT480 \\
\cline { 2 - 3 } & Outside diameter & $19.05 \mathrm{~mm}$ \\
\cline { 2 - 3 } & Nominal thickness & $2.77 \mathrm{~mm}$ \\
\cline { 2 - 3 } & tsr & $2.3 \mathrm{~mm}$ \\
\hline Drawing-out Speed & $150 \mathrm{~mm} / \mathrm{s}$ \\
\hline Rotating Speed & $12,000 \mathrm{rpm}$ \\
\hline Repeat frequency & $12,000 \mathrm{~Hz}$ \\
\hline \multirow{2}{*}{ Measurement pitch } & Cricumferential direction & $0.71 \mathrm{~mm}$ \\
\cline { 2 - 3 } & Longitudinal direction & $0.75 \mathrm{~mm}$ \\
\hline
\end{tabular}

Water is filled inside the heating tube and ultrasonic test equipment is inserted into it. It goes on along the center of a heating tube, it reflects right-angled by an ultrasonic reflective mirror, and the ultrasonic wave discharged from the ultrasound probe is equivalent to the inside of a heating tube. The ultrasonic wave reflected by the inside or the external surface of the heating tube returns the same course, and is detected by an ultrasound probe. In the case of actual measurement, it measures spirally, pulling a probe at the same time it rotates an ultrasonic mirror according to the power of a motor or water. An ultrasonic reflective mirror rotates with a high-speed rotating speed, and an ultrasonic wave is scanned in the circumferential direction in the inside of a heating tube by it. An ultrasound probe discharges an ultrasonic wave on predetermined repeat frequency .

Therefore, with rotation of an ultrasonic reflective mirror, and the combination of the repeated frequency of an ultrasonic wave, the measurement pitch of the circumferential direction becomes settled.Moreover, ultrasonic test equipment moves to the longitudinal direction of a heating tube at a predetermined drawing-out speed. With the combination of this drawing-out speed and the rotating speed of an ultrasonic reflective mirror, the measurement pitch of the shaft orientations of a heating tube becomes settled. Table. 1 shows the example of the specifications of tubes and the measurement pitch in the measurement condition.

Fig. 1 (b) shows the diagrammatic chart which shows typically the echo data when measuring one point ultrasonically. A horizontal axis is lapsed time from the discharge time of an ultrasonic wave. A vertical axis is echo height. Echo $\mathrm{T}$ is an echo reflected by the ultrasonic reflective mirror, Echo $\mathrm{S}$ is an echo reflected by the inside of the heating tube, and Echo B is an echo reflected by the outside of the heating tube. Fig. 1 shows the situation of these echoes T, S, and B typically. In Fig. 1 (b), the time lag of Echo S and Echo $\mathrm{B}$ is equivalent to wall thickness. This measured time lag is convertible into the wall thickness of a heating tube.

\subsubsection{Measurement data}

For this evaluation, not only the digital data of a minimum thickness value that receives per tube but the digital data of every measurement pitch is used. Fig. 2 shows example figures of the recorded data of wall thickness. The wall-thickness data of the every point of the heating tube measured by IUT is memorized by recording equipment. The number of data of wall-thickness data is extensive. For example, in the circumferential direction inside a heating tube, when it assumes that 50 points are measured per round and it measures in $0.75 \mathrm{~mm}$ pitch in axis. In this case of a heating tube $10 \mathrm{~m}$ in length, 667,000 wall-thickness data will be memorized to recording equipment on the whole. Fig. 2 shows the schematics of the recorded data in two dimensions. This figure is equivalent to distribution of the wall-thickness data when developing the inside of a heating tube at a plane. One division is equivalent to one measurement point, and wall-thickness data is attached to an every place point. The point shown by hatching is a point which was less than the threshold, and the 
point which it was black and was smeared away is a reappraisal point mentioned later.

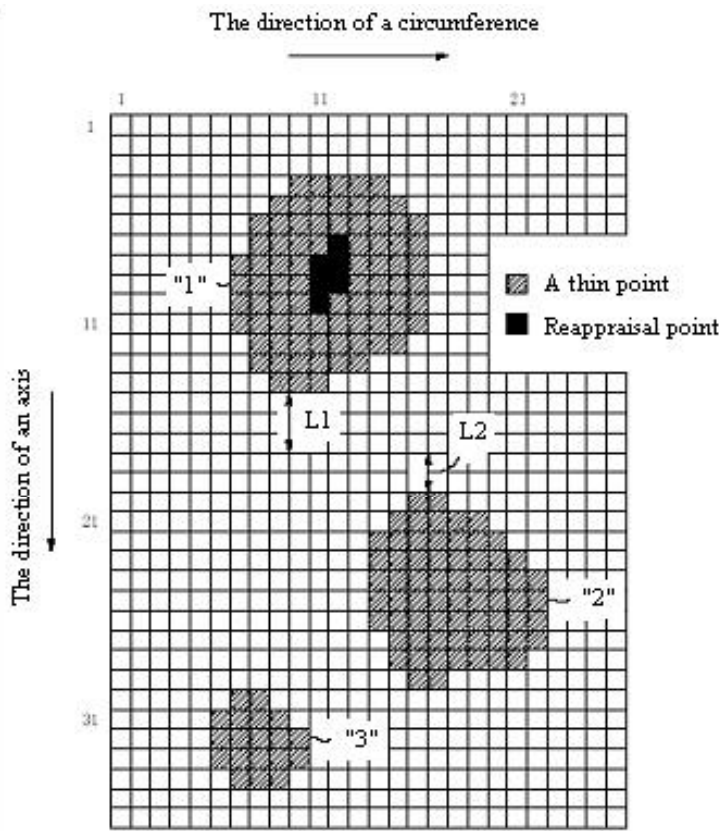

Fig. 2 Wall-thickness measurement record data array figure

\subsection{Inspection part}

The inspection range of a heating tube makes foundations from a tube end to the Bend part, and selection of the heating tube and position to measure is appropriately set up from a thinning tendency according to an internal structure or the state of steam and drain-pipe in-and-out.It is electing a sample tubing (it being about 5 to $10 \%$ to a whole number), and grasping the thinning tendency of tube arrangement and the direction of a tube axis, when a tendency's is unknown although carried out a principle [ the part which grasps the thinning tendency ]. In addition, a sample tubing decides to take in the shape of a lattice 4 or every 5 to pipe arrangement. Thinning and the corrosion generating part are as follows experientially [ the former ].

- A penetration part with a tube support plate (inside thinning by scale adhesion and exfoliation, outside thinning by drain attack)

- Steam inlet zone (outside thinning by steamy erosion)

- The tube-support-plate part near a steam inlet (outside thinning by drain attack)

- Drain cooling zone entrance tube-support-plate part (outside thinning by steamy erosion)

- Drain cooling zone (a drain attack, outside thinning by corrosion)

Although thinning may be looked at in a tube end weld zone by erosion and corrosion, it is not applicable in this evaluation.

\subsection{Evaluation methods}

Thinning point is determined comparing measured data with a required minimum thickness. A required minimum thickness is given by the following equation ${ }^{[2]}$.

$$
\mathrm{tsr}=\mathrm{PD} /\left(2 \sigma_{\mathrm{a}} \eta+0.8 \mathrm{P}\right) .
$$


where:

tsr: minimum required thickness of a steel tube $(\mathrm{mm})$,

$\mathrm{P}$ : maximum allowable working pressure $(\mathrm{MPa})$,

$\mathrm{D}$ : the modulus of outside-diameter of steel pipe ( $\mathrm{mm})$,

$\sigma_{\mathrm{a}}$ : allowable tensile stress of material at the operation condition( $\left.\mathrm{MPa}\right)$,

$\eta:$ the efficiency of longitudinal joint

The form of the evaluation method is consisted with local thinning evaluation (evaluation of the longitudinal direction, and evaluation of the circumferential direction), stress evaluation of a minimum thickness part, and pitting corrosion evaluation.

\subsection{Evaluation procedure}

Fig. 3 is a flow chart of the whole evaluation method.

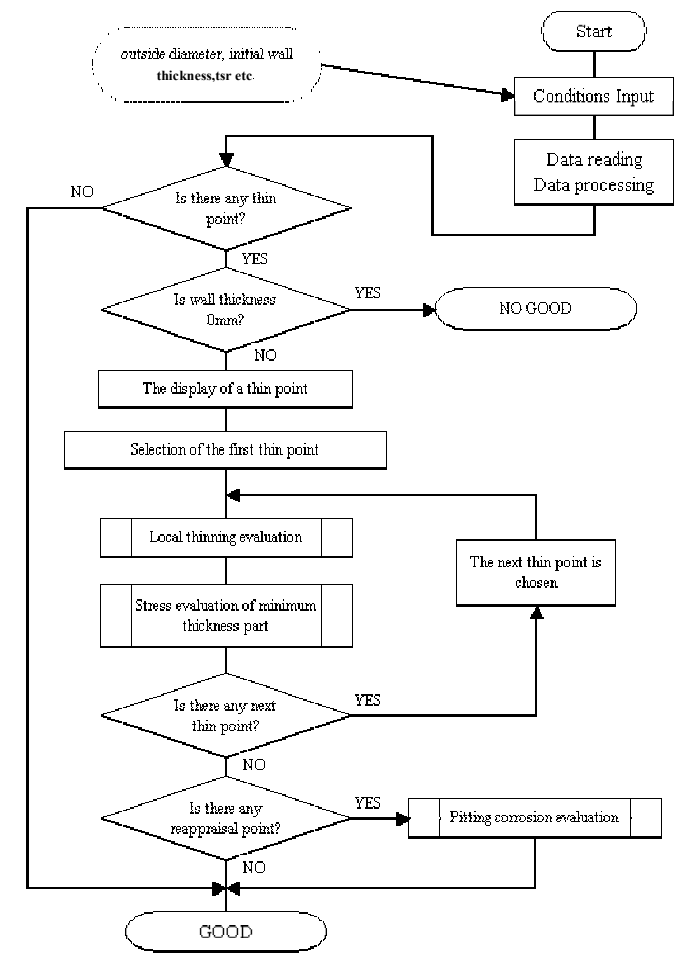

Fig. 3 Wall-thickness evaluation flow chart

Evaluation is started and conditions, such as the outside diameter, initial wall thickness of heating tube, and tsr are inputted first. Next, a lot of measurement data is read from memory storage. And wall-thickness data is put in order so that it may become arrangement as shown in Fig. 2. This is equivalent to "data processing". "Thin point" is defined to the point which is less than threshold (tsr). If wall thickness has exceeded the threshold at all the measurement points, a judgment is set to "NO" and an evaluation procedure will shift to "GOOD". If a thin point exists at least one, it will shift to subsequent procedures. This evaluation has the feature in extracting only a really dangerous situation by continuing an evaluation procedure, without considering it as "NO GOOD" immediately even if a thin point exists. In the judgment of a thin point, it will shift from whether wall thickness is $0 \mathrm{~mm}$ to a judgment about all the thin points. If set to "NO" by this judgment, it will shift to the following procedure and all the thin points will be displayed on a screen. The example of a display of a thin point is shown in Fig. 2. The point which performed hatching is a thin point. Next, the first thin point is chosen. "First thin point" is defined to the smallest number point both longitudinal and circumferential direction. And "local thinning evaluation" and 
"stress evaluation of a minimum thickness part" are performed at the thin point. In these evaluations, the wall-thickness data of the perimeter is also taken into consideration. And it is judged whether it is really dangerous or safe in strength. A dangerous thin point is memorized reappraisal point. After such work is completed about one thin point, it judges whether there is any next thin point. If the next thin point exists, the next thin point is chosen and "local thinning evaluation" and "stress evaluation of a minimum thickness part" are repeated. After completing local thinning evaluation and stress evaluation of a minimum thickness part for all the thin points, it is judged whether the reappraisal point was memorized. If a reappraisal point exists, it will move to "pitting corrosion evaluation". And pitting corrosion evaluation is performed about a thin domain including a reappraisal point, for judging whether it is really dangerous. If dangerous in "pitting corrosion evaluation", it will be judged "NO GOOD", and an evaluation procedure will be completed.

\section{Local Thinning}

\subsection{Local thinning evaluation}

Local thinning evaluation is divided into evaluation of the longitudinal direction, and evaluation of the circumferential direction. And evaluation of the longitudinal direction includes the evaluation based on the "length of longitudinal direction" for continuous thinning domain, and the evaluation by an area compensating method based on "the area of shortage domain ". This evaluation method is based on the structural theories as follows. Hole within half size (however, $508 \mathrm{~mm}$ or less) of the maximum outside diameter (outside diameter of $1520 \mathrm{~mm}$ or less) can usually be established in cylinder structure. And then the domain effective in reinforcement of a hole is taken to longitudinal direction as to the range of a hole diameter (1/2 of an outside diameter) from the hole center, respectively ${ }^{[3]}$.

\subsection{Evaluation of the longitudinal direction}

Continuous thin point of the longitudinal direction is specified for the evaluation. And it is judged whether the longitudinal direction range of a thin domain is settled within the outside diameter $\mathrm{D}$ of a heating tube centering on the thin point concerned about the thin domain including the thin point concerned. This is explained using the diagrammatic chart of Fig. 4. Fig. 4 (A) shows the schematic diagram which shows a part of distribution of the longitudinal direction of wall-thickness data. A horizontal axis is a position of the longitudinal direction and an axis of ordinate is wall thickness. The measurement pitch of longitudinal direction is $\mathrm{p} 1$. The black dot shows the wall-thickness data of the thin point concerned. ta is the initial wall thickness of a heating tube, and tsr is a wall-thickness threshold. The domain where the thin point continues continuously from black dot data is a thin domain. If this thin domain puts in another way whether it is settled within the outside diameter $\mathrm{D}$ centering on the thin point of a black dot, it will be judged whether it is settled within D (1/2) of the both sides of the longitudinal direction. If it is "NO", it is dangerous, and reappraisal will serve as a required thin point and the thin point of this black dot will memorize this thin point as a "reappraisal point." And local thinning evaluation is ended as it is. If set to "YES", it will shift to a judgment with the following area compensating method. 

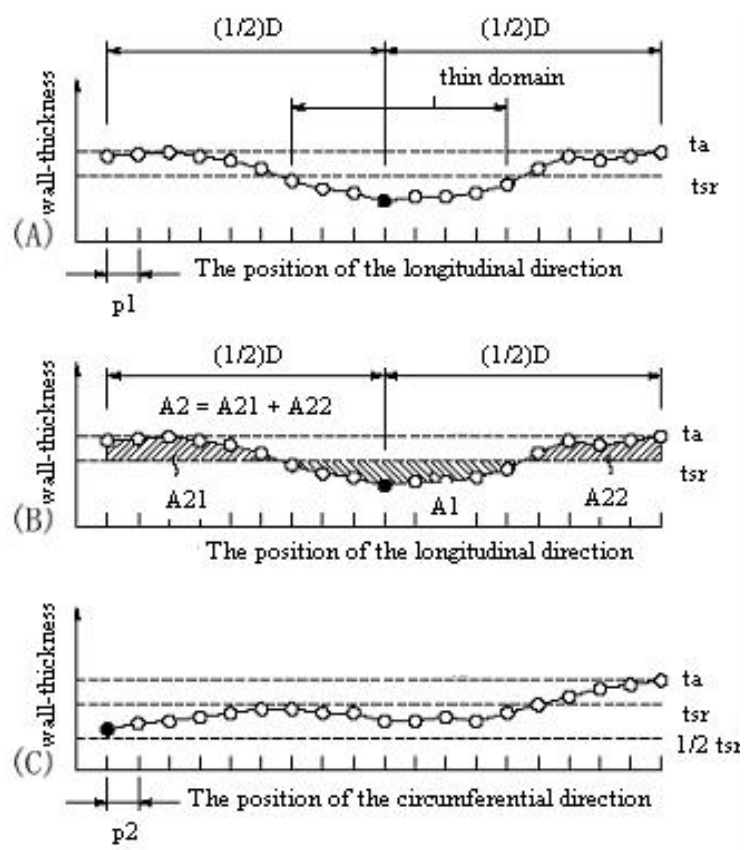

Fig. 4 Wall-thickness data both longitudinal and circumferential direction

An area compensating method evaluates a heating tube based on the cross-sectional area of the thin domain in the longitudinal direction section centering on a thin point. This is explained using the diagrammatic chart of Fig. 4 (B). Fig. 4 (B) shows distribution of the longitudinal direction of wall-thickness data like Fig. 4 (A). In this diagrammatic chart, the cross-sectional area of the thin domain in the longitudinal direction section is made an issue of. The area of the domain which is less than tsr is A1.In within the limits of the outside diameter $\mathrm{D}$ centering on the thin point of a black dot,the sum total of the cross-sectional area of the point which is not less than tsr is $\mathrm{A} 21+\mathrm{A} 22=\mathrm{A} 2$. It is judged whether $\mathrm{A} 1$ is smaller than A2. If it is "NO", it is dangerous, and reappraisal will serve as a required thin point and the thin point of this black dot will memorize this thin point as a "reappraisal point." And local thinning evaluation is ended as it is. If set to "YES", it will shift to evaluation of the next circumferential direction.

\subsection{Evaluation of the circumferential direction}

In evaluation of the circumferential direction, it is judged whether wall thickness is less than $1 / 2$ tsr at some point of the direction of a circumference in the direction section of a circumference (section perpendicular to the longitudinal direction of a heating tube) which includes the interruption point about the thin point concerned. This is explained using the diagrammatic chart of Fig. 4 (C). Fig. 4 (C) shows a part of distribution of the circumferential direction of wall-thickness data. A horizontal axis is a position of the circumferential direction, and a vertical axis is wall thickness. The measurement pitch of the circumferential direction is $\mathrm{p} 2$. The point of a black dot is the thin point concerned.

It is judged whether one wall thickness of the circumferential directions is less than 1/2 tsr. If less than neither of the measurement points, local thinning evaluation is ended.

In this case, local thinning evaluation will be ended, without the thin point in particular concerned not having a dangerous thing, and memorizing as a reappraisal point. Though wall thickness is less than $1 / 2$ tsr at the point of one of the circumferential directions, if there is wall thickness of an average of the circumferential direction of beyond a predetermined value, there will be no danger that strength is insufficient. Therefore, it is 
judged whether the wall thickness of an average of the circumferential direction is more than $1 / 2$ tsr. If a judgment is "NO", it is dangerous, and reappraisal will serve as a required thin point and the interruption point concerned will memorize this thin point as a "reappraisal point." And local thinning evaluation is ended.

If a judgment is"YES",without there being no dangerous thing and memorizing as a reappraisal point, especially the thin point concerned will end local thinning evaluation, and it will shift from it to stress evaluation of a minimum thickness part.

\section{Stress Evaluation of Minimum Thickness Part}

The stress concerning a thinning part decreases because the part of thick wall thickness distributes. The difference from this load to the wall-thickness minimum part and equivalent thickness is removed with an internal pressure to all the wall-thickness measured regions, and, specifically, it calculates by achieving equalization of a stress.

\subsection{Stress evaluation procedure of minimum thickness part}

Since the thin domain is already pinpointed as explained in Fig. 4(A), the point which has the minimum wall-thickness data in the thin domain is a minimum thickness part. First, in the longitudinal direction of a tube, within the limits of the outside diameter D centering on a minimum thickness part is divided into two or more evaluation domains. This is explained using the diagrammatic chart of Fig. 5(A).

Fig. 5(A) shows the diagrammatic chart which shows a part of distribution of the longitudinal direction of wall-thickness data. A horizontal axis is a position of longitudinal direction and a vertical axis is wall thickness. The black dot shows the wall-thickness data of the minimum thickness part. The domain of the outside diameter $\mathrm{D}$ within the limits is pinpointed focusing on this minimum thickness part. And longitudinal direction of wall-thickness data divides within the limits of the outside diameter D into two or more domains by making into a boundary point the point which crosses the wall-thickness threshold tsr.

It is divided into "a" domain, "b" domain, and "c" domain in Fig. 5 (B). "b" domain is a domain in which wall thickness is less than tsr, and "a" domain and "c" domain of the both sides are a domain where wall thickness exceeds tsr.
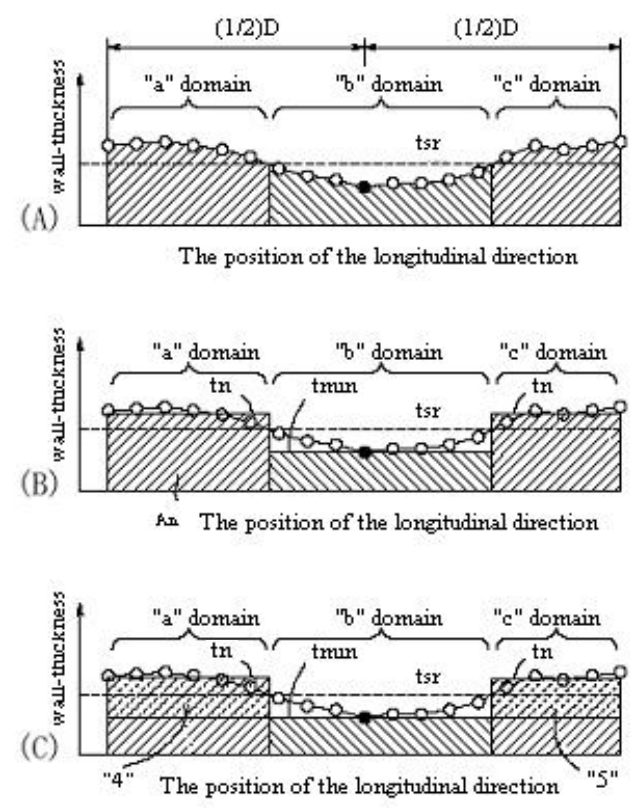

Fig.5 Minimum thickness part stress evaluation diagram 
Next, the stress $\sigma_{\mathrm{n}}$ of each evaluation domain is computed. The average value of wall thickness is set to $t_{n}$ in $n$ domain. Stress $\sigma_{n}$ in $n$ domain is calculable by the following equation $^{[2]}$.

$$
\sigma_{\mathrm{n}}=\mathrm{P}\left(\mathrm{D}-0.8 \mathrm{t}_{\mathrm{n}}\right) /\left(2 \mathrm{t}_{\mathrm{n}}\right)
$$

where:

$\mathrm{P}$ : maximum allowable working pressure applied inside a tube( $\mathrm{MPa})$,

D: the outside diameter of a tube $(\mathrm{mm})$,

$t_{n}$ : the average value of the wall thickness of " $n$ " domain(mm)

$\sigma_{\mathrm{n}}$ is computed regarding " $\mathrm{b}$ " domain which is thinner than tsr, using the minimum thickness $t_{\min }$ as $t_{n}$ in equation(2).

Next, the load $\left(F_{n}\right)$ of each evaluation domain is computed. Load $\left(F_{n}\right)$ is calculable by the following equation.

$$
\mathrm{F}_{\mathrm{n}}=\sigma_{\mathrm{n}} \cdot \mathrm{A}_{\mathrm{n}}
$$

where:

$\sigma_{\mathrm{n}}$ : the stress computed by equation $(2)(\mathrm{MPa})$,

$\mathrm{A}_{\mathrm{n}}$ : the cross-sectional area of each evaluation domain $\left(\mathrm{mm}^{2}\right)$

As shown in Fig. 5 (B), the cross-sectional area $A_{n}$ of "a" domain can multiply by them and find the average value $t_{n}$ of wall thickness, and the length of the longitudinal direction of "a" domain. "c" domain is also the same. Regarding "b" domain, it multiplies by the minimum thickness $t_{\min }$ and the length of the longitudinal direction of " $b$ " domain, and is considered as the cross-sectional area $A_{n}$.

Next, the corrected load $F_{n m}$ on the basis of the minimum thickness $t_{\min }$ is computed for each evaluation domain. The corrected load $\mathrm{F}_{\mathrm{nm}}$ is calculable by the following equation.

$$
\mathrm{F}_{\mathrm{nm}}=\mathrm{F}_{\mathrm{n}}\left(\mathrm{t}_{\min } / \mathrm{t}_{\mathrm{n}}\right)
$$

This corrected load is equivalent to the load which deducted the load of the cross-sectional-area domains of " 4 " and " 5 " shown by hatching of the dashed line from $F_{n}$ as illustrated in Fig.5 (C). The stress redistrtibution is taken into consideration which is occurred by the connection of each domain where each corrected load derived by equation(4) is applied. The redistributed applied stress $\sigma_{1}$ in the connected minimum thickness $t_{\min }$ domains can be evaluated in the safety side by the following equation.

$$
\sigma_{1}=\left(\sum \mathrm{F}_{\mathrm{nm}}\right) /\left(\mathrm{t}_{\min } \cdot \mathrm{D}\right)
$$

In equation(5), the numerator of the right-hand side totals the corrected load $\mathrm{F}_{\mathrm{nm}}$ of each domain. The denominator of the right-hand side is the area of the component of the thickness of $t_{\min }$. Then the applied stress $\sigma_{1}$ is judged whether it is less than the allowable tensile stress $\sigma_{\mathrm{a}}$ of the material. If the judgment is "NO", it will be dangerous and this minimum thickness part will be memorized as a "reappraisal point." If the judgment is "YES", the concerned local thinning is evaluated as acceptable.

\section{Pitting Corrosion Evaluation}

Pitting corrosion is evaluated by the theory of an independent hole, which is not based on 
stress evaluation andwhen the path of a hole is $1 / 4$ or less of a pipe bore, JSME Code rules $^{[3]}$ show that reinforcement of a hole is not needed. In the case of pitting corrosion, the residual lifetime until the leak before break is evaluated. Pitting corrosion evaluation consists of two step procedures.

The first step of the pitting corrosion evaluation is to judge whether the concerned reappraisal domain is independent or not.

In the case of pitting corrosion evaluation, one reappraisal point exists at least. First, the thin domain (this is defined as a reappraisal domain) containing one reappraisal point at least is created. This reappraisal domain will be created for all the memorized reappraisal points. In Fig. 2, the thin domain including a reappraisal point is shown and this is a reappraisal domain. Since the thin domain of others which are shown in Fig. 2 does not include the reappraisal point, it is not a reappraisal domain. First, the first reappraisal domain is chosen. Next, the range L effective in reinforcement of a hole is computed for the reappraisal domain concerned. Moreover, the range $\mathrm{L}$ effective in reinforcement of a hole is computed also for the thin domain of the next door of the reappraisal domain concerned. This is explained with reference to Fig. 6 (A).

Fig. 6 (A) is an axial sectional view of a heating tube, and a hole exists in the inside of a heating tube in this drawing. This hole is recognized by distribution of the longitudinal direction of wall-thickness data. It asks for the cross-sectional area A3 of the portion which is less than tsr among the cross-sectional form of this hole. Next, range $\mathrm{L}$ is computed based on equation(6).

$$
\mathrm{L}=(\mathrm{A} 3 / 2) /\left(\mathrm{t}_{\mathrm{a}}-\mathrm{tsr}\right)
$$

(A)

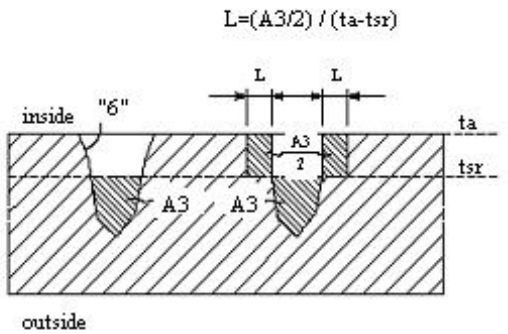

(B)

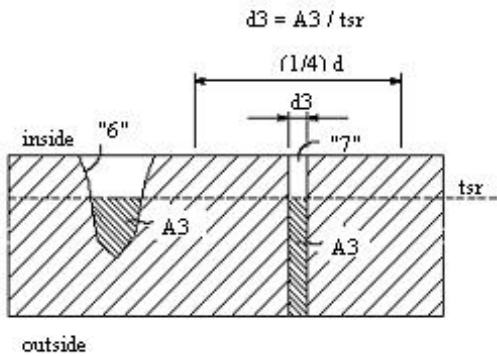

Fig.6 Equivalent hole diameter diagram

The meaning of range $\mathrm{L}$ is as follows. When the cross-sectional area A3 of the portion which is less than tsr is assumed to reinforce with "the portion which is not less than tsr" of the perimeter, the range $\mathrm{L}$ of a reinforcement portion is set that the cross-sectional area of the sum total of the reinforcement portion is set to A3. That is, it is a view which is going to reinforce it with "the portion which is not less than tsr" around a hole to the portion of the hole which is less than tsr and is weak. If "the portion which is not less than tsr" fully spreads to the perimeter of a hole and it goes into it, it will be judged that this hole is satisfactory in intensity. Therefore, when the next hole exists near this hole, it becomes 
important that the reinforcement range $\mathrm{L}$ of this hole and the reinforcement range of the next hole do not overlap. It is equivalent to judging whether a hole is "an independent hole." In Fig. 2, the range L1 effective in reinforcement of this reappraisal domain 1 is shown in the lower part of the reappraisal domain 1. Moreover, the range L2 effective in reinforcement of this thin domain 2 is shown in that upper part about the thin domain 2 of the next door of the reappraisal domain 1. If ranges L1 and L2 have overlapped, it will be "it being unusual and ending." An evaluation procedure is completed at the time. If it has not overlapped, it shifts to the judgment of the following equivalent pore size. In addition, in the above-mentioned explanation about the existence of an overlap of ranges L1 and L2, although only the existence [ the longitudinal direction of a tube ] of the overlap was explained, the existence of an overlap of ranges L1 and L2 is judged in fact also about the circumferential direction and the direction of slant (direction which makes the angle of 45 degrees to direction of an axis and the circumferential direction) of a tube.

The second step of the pitting corrosion evaluation is to calculate the equivalent pore size.

It is judged about the reappraisal domain concerned whether the equivalent pore size is below $1 / 4 \mathrm{~d}$. This is explained with reference to Fig. 6 (B). It asks for the cross-sectional area $\mathrm{A} 3$ of the portion which is less than tsr among the cross-sectional form of a hole 6 in the axial sectional view of a heating tube. Next, the penetration hole 7 of the shape of a straight line from which the cross-sectional area of the portion below tsr is set to A3 is assumed.

This penetration hole 6 is an equivalent hole of a hole 7. The pore size of this equivalent hole is set to $\mathrm{d} 3$. These $\mathrm{d} 3$ can be calculated by the following equation.

$$
\mathrm{d} 3=\mathrm{A} 3 / \mathrm{tsr}
$$

It is judged whether this equivalent pore size $\mathrm{d} 3$ is $1 / 4$ or less of the bore $\mathrm{d}$ of a heating tube. If an equivalent pore size $\mathrm{d} 3$ is $1 / 4$ or more of a hole $\mathrm{d}$, it will be "it being unusual and ending." An evaluation procedure is completed at the time. If an equivalent pore size $\mathrm{d} 3$ is smaller than $1 / 4$ of the bore diameter, it will be judged whether there is any next reappraisal domain. If there is the next reappraisal domain, the next reappraisal domain will be chosen and it will return to calculation of the range L effective in reinforcement of a pore. If there is no next reappraisal domain, pitting corrosion evaluation will be ended. In this case, it is "it being normal and ending."

As for the evaluation of pitting corrosion, residual life-time management until pitting corrosion penetrates the wall thickness can be performed based on the wall thinning rate data stored separately.

\section{Validity of the Proposed Method}

Practical inspection and evaluation has been performed to verify the validity of the proposed method. Table 2 summarizes a typical example of the inspection condition and the evaluation result of 188 tubes.

According to the most conservative criterion, if the wall thickness measurement data of a tube contain the thickness data less than tsr, plugs were installed at the both ends of the concerned tubes, the number of which was 38 in the case listed in Table2. However, in the proposed method, local thinning evaluation and stress evaluation were performed for 38 reappraisal tubes to be evaluated as acceptable for 20 tubes. It has been confirmed that the application of this method realized the advanced rationality on the maintenance technology of the feed water heater tubes. 
Table 2 Example of the inspection condition and the evaluation result

\begin{tabular}{|l|c|c|}
\hline & GOOD & NO GOOD \\
\hline Assessment by tsr & 150 & $\ddots \because 38 \because \ddots$ \\
\hline Local Thinning Assessment & $\therefore 23 . \therefore$ & 15 \\
\hline Stress Assesment & 20 & $\because \because 3 \cdot \because \because$ \\
\hline Pitting Assessment & 0 & 3 \\
\hline \hline Assessment Result & 170 & 18 \\
\hline
\end{tabular}

\section{Conclusion}

(1) The evaluation method of the tube was developed which evaluates synthetically the wall-thickness data of two or more points, and can judge automatically whether a tube is accepted or not.

(2) This system can realize the shortening of large evaluation time and reduction of repair expense.

\section{References}

[1] Hokkaido machine industry society-the No. 27 inspection technical convention P7-11

[2] JIS B 8201-2005 Sationary steel boilers-Construction

[3] JSME S TA1-2003 Rules on Thermal Power Generation Facilities (PG-32.3.2,PG-36.2.1, PG32.1.3.1) 